\title{
Water oxygenation by fluidic microbubble generator
}

\author{
V Tesař ${ }^{1}, K$. Peszynski $^{2}$ \\ ${ }^{1}$ Institute of Thermomechanics v.v.i., Academy of Sciences of Czech Rep., 18200 Prague, Dolejškova 5, Czech Republic \\ ${ }^{2}$ University of Technology and Life Sciences in Bydgoszcz, 85789 Bydgoszcz, Kaliskiego 7, Poland
}

\begin{abstract}
Oxygenation of water by standard means in waste water processing, in particular to improve the conditions for the micro-organisms that decompose organic wastes is rather ineffective. The classical approach to improvements - decreasing the size of the aerator exits - have already reached their limits. A recent new idea is to decrease the size of the generated air bubbles by oscillating the supplied air flow using fluidic oscillators. Authors made extensive performance measurements with an unusual high-frequency fluidic oscillator, designed to operate within the submersed aerator body. The performance was evaluated by the dynamic method of recording the oxygen concentration increase to saturation in the aerated water. Experiments proved the fluidic generator can demonstrably increase the aeration efficiency 4.22-times compared with the aeration from a plain end of a submerged air supply tube. Despite this significant improvement, the behaviour of the generator still provides an opportunity for further improvements.
\end{abstract}

\section{Introduction}

Water under usual conditions contains dissolved oxygen - a fact of essential importance for aquatic organisms, the very life of which depends on sufficiently high oxygen concentration. Low oxygen contents is the main problem in waste water processing, in which organic wastes are decomposed by aquatic micro-organisms. Under low oxygen concentration conditions they cannot perform the task or even die and do not exist there at all. This is why a very important part of the waste water processing is aeration. It may be applied in several different forms (weirs, rotating cylinders with paddles or bristles, bubbling compressed air from an aerator, ...) all of which are relatively expensive, so that on their operation is spent the main proportion of processing costs.

A promising efficient method recently developed [1] is to blow through the aerator holes a pulsating air flow generated in a fluidic oscillator. It is possible generate this way microbubbles of sub-millimetre size, with extremely large total oxygen diffusion transfer surface while the oscillator device is inexpensive, reliable, maintenance-free, needs no electricity and consumes only little supplied pressure energy. In the standard version of applying this method, the aerator is submerged at the bottom of the water processing vessel while the oscillator has been usually located at the compressed air inlet above the vessel. The oscillation frequency has to be rather high - in resonance with the natural frequency of the microbubbles, which is of the order of $\mathrm{kHz}$. Such highfrequency oscillation, however, tends to be strongly damped in the tubes connecting the oscillator with the aerator.
As a remedy is now introduced an integral unit containing both parts (the oscillator as well as the aerator) in a single fully submersible body [2]. This paper describes an example of such a unit and provides a detailed description of the used method of evaluating its performance from the measured rate of water oxygenation.

\section{The generator}

The generator unit used in the experimental investigations discussed in this paper has its both parts, a no-moving-part fluidic oscillator on one hand and a pair of aerators on the other hand, integrated into a single stainless steel body measuring $273 \mathrm{~mm}$ x $250 \mathrm{~mm}$ in ground plan. The cavities for air flows were laser-cut in a rather thin ( $2 \mathrm{~mm}$ thickness) main plate, shown in Figure. 1. They are closed from above as well as from below by thicker $(8 \mathrm{~mm})$ cover plates.

Both parts of the generator are somewhat unusual. The oscillator consists, as usual, of a fluidic amplifier and feedback elements. The amplifier is of the jet-deflection diverter type. There are, however, no Coanda-effect attachment walls and the feedback elements are not the signal carrying channels one would expect to connect the amplifier output terminals with the control inputs. Instead, there are two relatively large (but shallow) cavities on both sides of the splitter wedge, the apex of which is positioned opposite to the exit from the supply nozzle S (Figure. 2). The feedback action is provided by vortical motions in these cavities (Figure. 1). A 
.photograph of the main plate of the integrated unit is shown in Figure. 3.

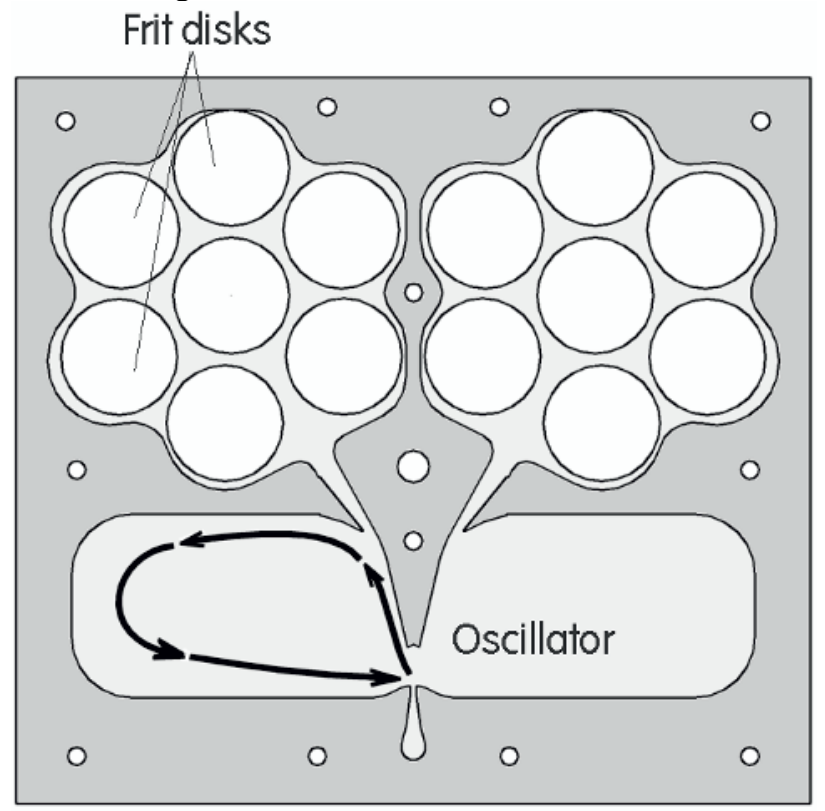

Figure 1. The investigated integral unit (oscillator + aerator) is made from three stainless-steel plates. This drawing shows the thin main plate ( $2 \mathrm{~mm}$ thickness) with working cavities made by laser cutting. The main plate is clamped between two thicker $(8 \mathrm{~mm})$ cover plates. Oscillator cavities are in the lower part of this picture, while the aerator manifold cavities are in the upper part. Air leaves the generator through tiny pores in 14 frit disks inserted into the top cover plate.

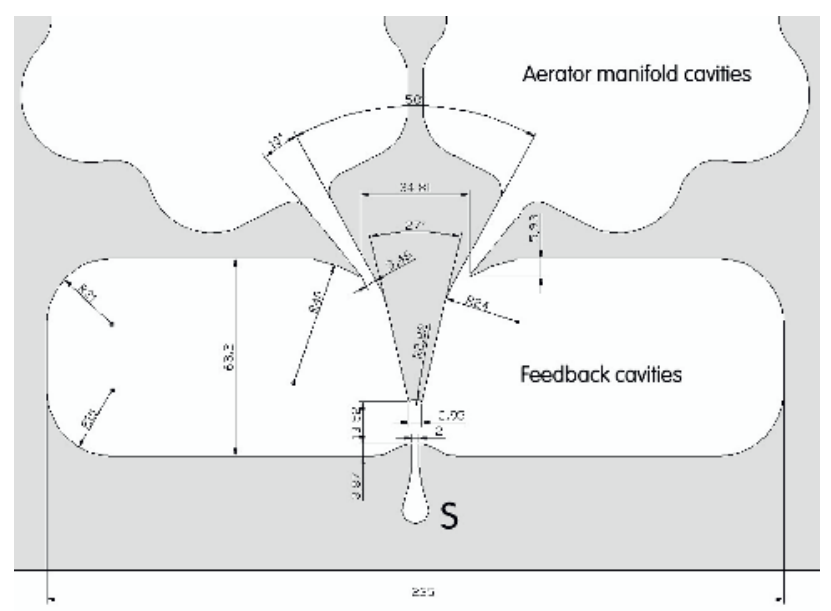

Figure 2. Geometry and dimensions of the fluidic oscillator that delivers alternating air flow pulses into the two aerator cavities. This unusual oscillator configuration has no feedback channels - their action is here replaced by vortical motions inside the feedback cavities (as is suggested by estimated flow pathlines at the left part of Figure 1). The advantage of this layout is very high achievable oscillation frequency - in the experiment discussed here it was $1.8 \mathrm{kHz}$.

There are two mutually independent aerators, each connected by a channel to one side of the splitter. They occupy the top part of the pictures Figures. 1 and 3. Each aerator consists of a quite large (but again shallow) manifold chamber which distributes the flow into a system of exits - represented by the pores in disks made of glass frit. When the oscillator oscillates, pulses of air flow are admitted in an alternating manner to one of the two manifolds. From there, the pulsating air flow passes into the water through pores in either of the two sets of 7 frit disks. The disks are of $40 \mathrm{~mm}$ diameter and $4 \mathrm{~mm}$ thickness. The frit material is sintered glass dust of particles guaranteed by the manufacturer (Kavalier Glass Works, Sázava) to be within a rather narrow range of dimensions. The pores for air flow between the particles are of widths from $40 \mu \mathrm{m}$ to $100 \mu \mathrm{m}$. Photograph of the assembled generator seen from above, with the frit disks in their positions, is presented in figure 4 .

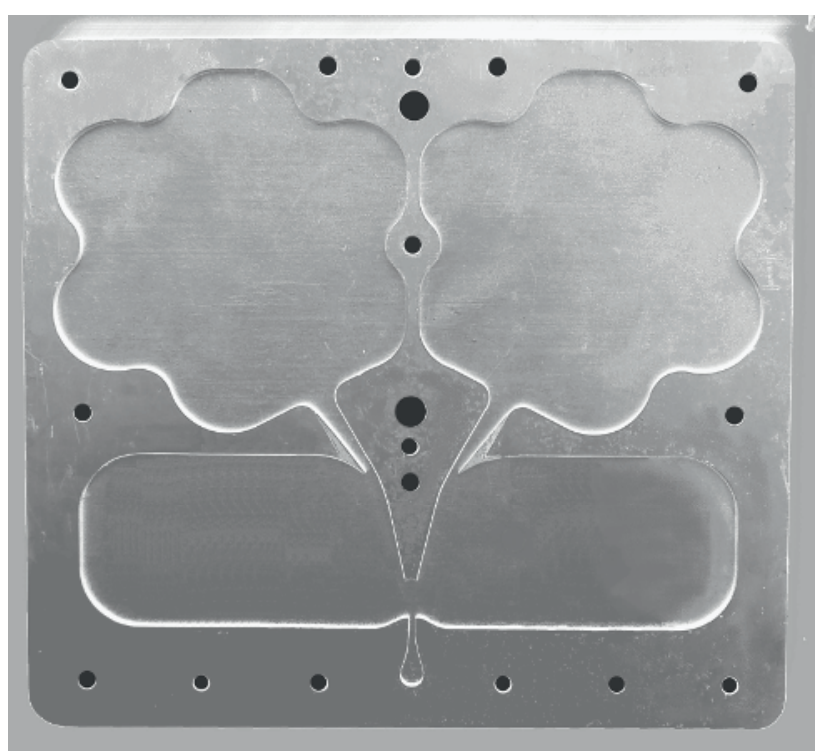

Figure 3. Photograph of the main plate with the laser-cut cavities. Also seen under the main plate is the bottom cover plate. The cavities are very shallow so that it is surprising that oscillatory motions are not damped by the inevitable fluid friction.

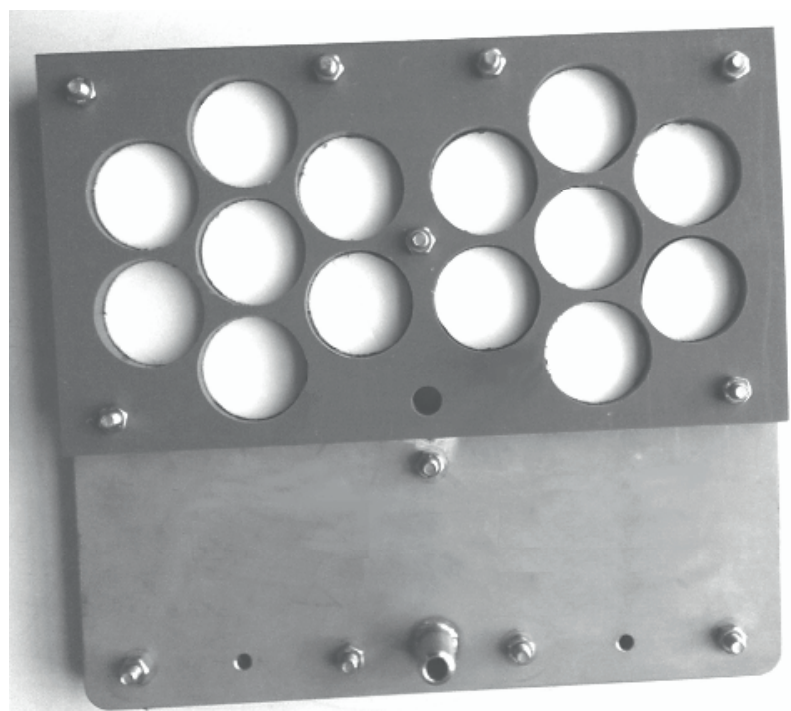

Figure 4. Photograph of the assembled integral unit, seen from above. In the 14 large holes of $40 \mathrm{~mm}$ diameter are positioned disks of glass frit, the pores in which serve as the aerator exits. 


\section{Basic facts about oxygen diffusion into water}

The diffusion transport may be analysed by considering the idea of the infinitesimal element presented in Figure. 5 - from reference [4] in which may be found additional explanations. Into the shown element of water (for simplicity rectangular shaped), of mass $\mathrm{M}[\mathrm{kg}]$, is transported oxygen $\mathrm{O}_{2}$ in the direction of the axis $X_{1}$ through the surface $F\left[\mathrm{~m}^{2}\right]$. The transport has to cross the boundary layer of thickness $\delta$. This acts as a resistance, opposing the transport.

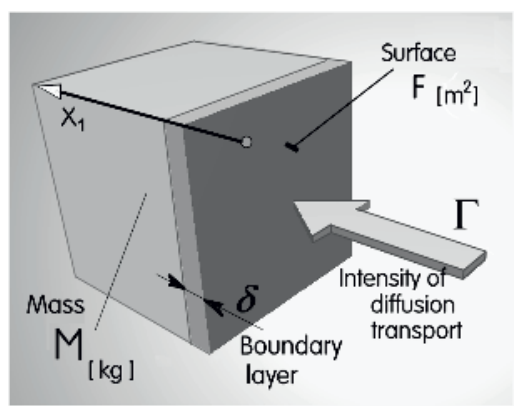

Figure 5. The basic concepts of diffusion transport the direction of the axis $X_{1}$ through the boundary layer across the surface area $F$ into the mass $M$ of water-a process governed by the Fick's law.

As the liquid(water) is oxygenated, the concentration of oxygen $\mathrm{m}_{\mathrm{O} 2}$ in it increases - and hence increases also the absolute mass $M_{\mathrm{O}_{2}}$ of the oxygen there. The concentration is defined

$$
m_{\mathrm{O}_{2}}=M_{\mathrm{O}_{2}} / M
$$

in the course of an incremental time interval $\mathrm{d} t$ the transported oxygen mass is $\mathrm{d} M_{\mathrm{O}_{2}}$, so that the intensity of transport $\Gamma\left[\mathrm{kg} /\left(\mathrm{m}^{2} \cdot \mathrm{s}\right)\right]$ may be defined

$$
\frac{\mathrm{d} M_{O_{2}}}{\mathrm{~d} t}=\Gamma F
$$

- where $F\left[\mathrm{~m}^{2}\right]$ is the area of the surface over which the oxygen enters the liquid. The corresponding rate of increase of the concentration is

$$
\frac{\mathrm{d} m_{\mathrm{O}_{2}}}{\mathrm{~d} t}=\frac{\Gamma F}{M}
$$

The law that governs the transport by gradient diffusion is the Fick's first law (the negative sign expresses the fact that the transport is directed opposite to the local concentration gradient) :

$$
\Gamma v=-\frac{\mathrm{d} m_{\mathrm{O}_{2}}}{\mathrm{~d} X_{1}} \chi
$$

- where $v\left[\mathrm{~m}^{2} / \mathrm{kg}\right]$ is specific volume of the liquid and $\chi\left[\mathrm{m}^{2} / \mathrm{s}\right]$ is introduced as the coefficient of mass transfer rate.
Inserting eq. (4) into eq. (2) leads to the basic expression of the dynamics of diffusion (of course, in only one-dimensional case - a more general form for three-dimensional flows is presented in [4])

$$
M \frac{\mathrm{d} m_{\mathrm{O}_{2}}}{\mathrm{~d} t}=\frac{\mathrm{d} M_{\mathrm{O}_{2}}}{\mathrm{~d} t}=-\frac{\mathrm{d} m_{\mathrm{O}_{2}}}{\mathrm{~d} X_{1}} \frac{\chi F}{v}
$$

Since the mass of transported oxygen $M_{\mathrm{O}_{2}}[\mathrm{~kg}]$ in the discussed experiments is very small (- typically the diffused amounts are only grams of oxygen $\mathrm{O}_{2}$ per cubic metre $\mathrm{m}^{3}$ - or, in other words, per ton of water) the value $M$ remains practically constant during the diffusion process. It is thus possible to simplify the analysis by considering $M$ a constant, equal to the mass of the water - and also as a constant will be considered the specific volume $v$ of water.

Despite its generality and importance, eq. (5) is unfortunately not really helpful for the present case of investigating the aeration by air bubbles - especially at these initial stages of investigation in which there is no available information about the quantities $F$ and $\chi$. After all, for the purposes pursued in this paper it is not necessary to investigate the spatial distribution of concentration inside the boundary layer. It is therefore useful to introduce another simplification: we shall assume a constant gradient in the boundary layer

$$
\frac{\mathrm{d} m_{\mathrm{O}_{2}}}{\mathrm{~d} X_{1}}=\frac{\Delta m_{\mathrm{O}_{2}}}{\delta}
$$

- so that with a suitable choice of the order of subtraction in evaluating the difference $\Delta m_{\mathrm{O}_{2}}$ the law eq. (5) is converted into the simplified expression

$$
\frac{\mathrm{d} m_{\mathrm{O}_{2}}}{\mathrm{~d} X_{1}}=\frac{\Delta m_{\mathrm{O}_{2}}}{\delta} \frac{\chi F}{v M}
$$

This, of course, means an introduction of yet another unknown quantity, the boundary layer thickness $\delta[\mathrm{m}]$. To measure it would lead to the problem of how to measure a distance between extreme points that are both in infinity (the concentration approaches on both sides of the layer an asymptotic value). In fact, the knowledge of $\delta$ would no bring anything useful to the analysis of performed experiments. It is therefore acceptable to introduce a summary auxiliary variable $\alpha_{m}[\mathrm{~m} / \mathrm{s}]$, which contains all the unknown variables - with the only exception of the surface area $F-$ and to write

$$
\frac{\mathrm{d} m_{\mathrm{O}_{2}}}{\mathrm{~d} t}=\alpha_{m} \kappa F \Delta m_{\mathrm{O}_{2}}
$$

- where the auxiliary constant variable $\kappa\left[\mathrm{m}^{-3}\right]$ is needed solely for dimensional reasons. The reason for the dimension $[\mathrm{m} / \mathrm{s}]$ of the mass diffusion coefficient $\alpha_{m}$ is the fact that in those cases (not treated here) where it is possible to evaluate the unknown variables, it should be useful to investigate the Sherwood number [4] defined 


$$
\mathrm{Sw}=\frac{\alpha_{m} \delta}{\chi}
$$

\section{Experiments}

The oxygenation experiments were made with the bubble generator unit submerged under the water surface in a glass-walled vessel of dimensions $0.212 \mathrm{~m} \times 0.342 \mathrm{~m}$, filled prior to introduction of the generator with distilled water to the height $0.190 \mathrm{~mm}$ above the bottom. The vessel thus contained $13.8 \mathrm{~kg}$ of water.

In the experiments discussed here, the supplied air flow into the generator was $0.611 \mathrm{~g} / \mathrm{s}$. Considering the usually given composition of atmospheric air as containing $21 \%$ of oxygen, the latter was supplied at the rate $0.128 \mathrm{~g} / \mathrm{s}$.

The flow rate was measured by electronic mass flowmeter FMA1827 (manufactured by OMEGA Engineering, Inc.) capable of measuring the flow rate up to $50 \mathrm{~g} / \mathrm{min}$, i.e. $0.83 \mathrm{~g} / \mathrm{s}$. Oscillation frequency was measured by an electret microphone using the audio card in the computer and a software performing the fast Fourier transformation. The basic frequency of the jet switching inside the oscillator was apparent in the spectrum as an immediately recognisable peak - its frequency was $1.8 \mathrm{kHz}$. It should be said that the oscillator is still in development and at the stage reported in this paper we were not yet fully satisfied with the intensity of the generated acoustic signal.

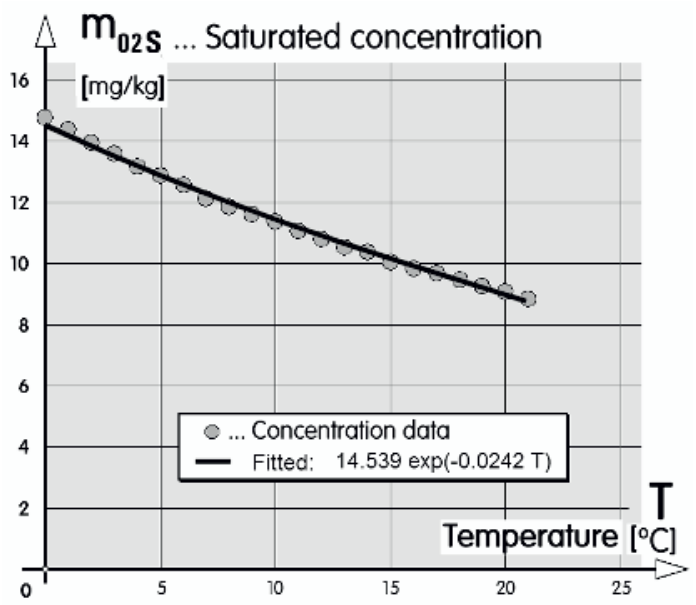

Figure 6. Concentration $m_{\mathrm{O}_{2}}$ of dissolved oxygen in water can reach at most the value $m_{\mathrm{O}_{2} \mathrm{~S}}$ (in the saturated state) which strongly depends on water temperature. Data are from ref. [15]. The exponential function was fitted for evaluations of measured data values in the discussed investigations.

The dissolved oxygen concentration in the water was measured by a probe supplied as "D.O.Kit" by Atlas Scientific, Brooklyn, USA. The electronic circuitry for processing the probe signal was built by Mr. M. Pavelka at the Institute of Thermomechanics. The values indicated by the probe were labelled as milligrams per litre obviously (because of the assumed constant value of water specific volume) equal to the instantaneous dimensionless mass concentration $m_{\mathrm{O}_{2}}$

The maximum concentration of dissolved oxygen in water is limited by saturation, which is strongly

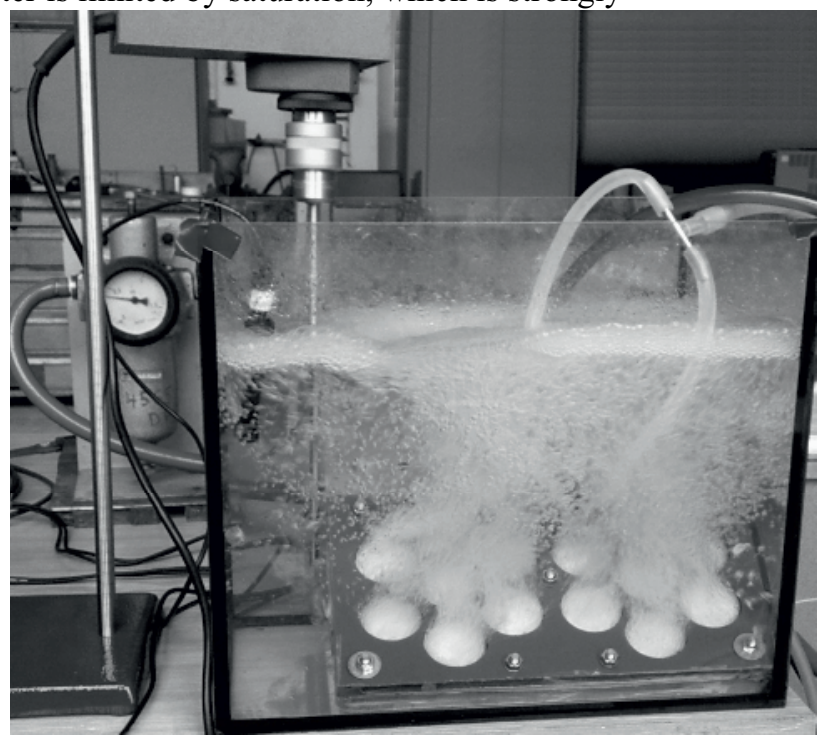

Figure 7. Photograph of the unit undergoing the test run with inactive oscillator - inside the fish-tank vessel. At left, inserted from above, is a propeller mixer that was run throughout all the tests.

dependent on water temperature, as shown in the diagram Figure. 6 from [15]. We have fitted the values with exponential function useful for data handling:

$$
m_{\mathrm{O}_{2} \mathrm{~S}}=14.539 \exp (-0.0242 T)
$$

Before each experimental run, the dissolved oxygen from the water in the vessel was removed by addition of sodium sulphite, with cobalt chloride as a catalyst. These additives were mixed by the propeller mixer seen in Figure. 7 until the concentration probe indicated zero concentration (with some additional waiting time). The mixer was left running throughout all experiments, even though the water was quite vehemently agitated by the generated bubbles.

\section{Data processing}

Of the several obtained measurement results, two very typical cases are here presented. Both were made at the above mentioned oxygen supply flow rate $0.128 \mathrm{~g} / \mathrm{s}$ (through the air inlet). In the first experimental run, the air was supplied into the submerged generator. In the subsequent comparison case, the air was introduced through a submerged open end of a $7 \mathrm{~mm}$ inner diameter tube, which reached $20 \mathrm{~mm}$ above the vessel bottom. In both cases, the sampling rate was $5 \mathrm{~s}$ between readings.

With the fluidic generator, the measurement running up to a reasonable approach to the saturated concentration lasted about 10 minutes. With the pain tube end, the generated bubbles were very powerful and produced strong waves on the water surface. This agitation produced an impression that there must be also a severe aeration. It was therefore quite surprising that the 
progress of oxygenation was much slower and the measurement run lasted for more than 1 hour.

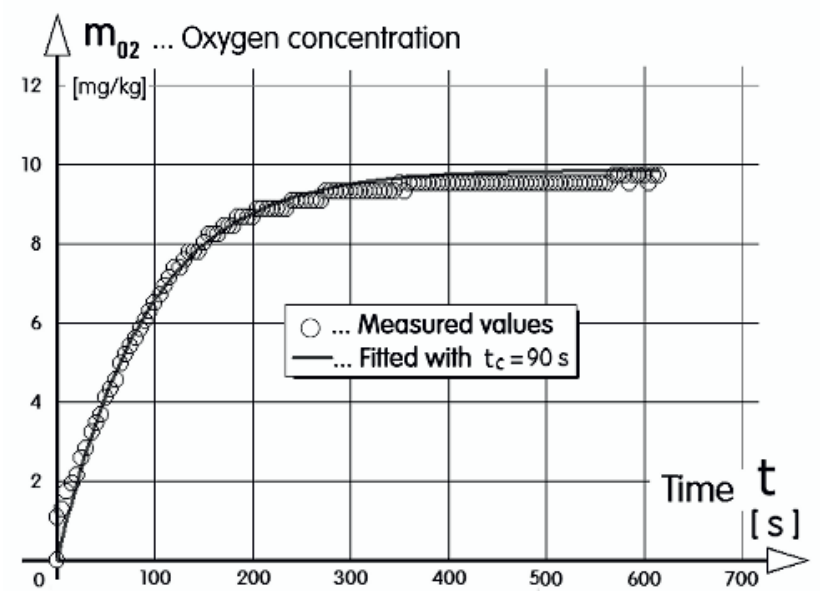

Figure 8. Oxygen concentration $m_{\mathrm{O}_{2}}$ in the water inside the aerated vessel. Measured at progressively increasing time (at $5 \mathrm{~s}$ intervals) - after the start of bubble generation (prior to which the oxygen was removed by chemical reaction). The supplied air flow rate was $0,61 \mathrm{~g} / \mathrm{s}$, frequency of oscillation $f=1.8 \mathrm{kHz}$.

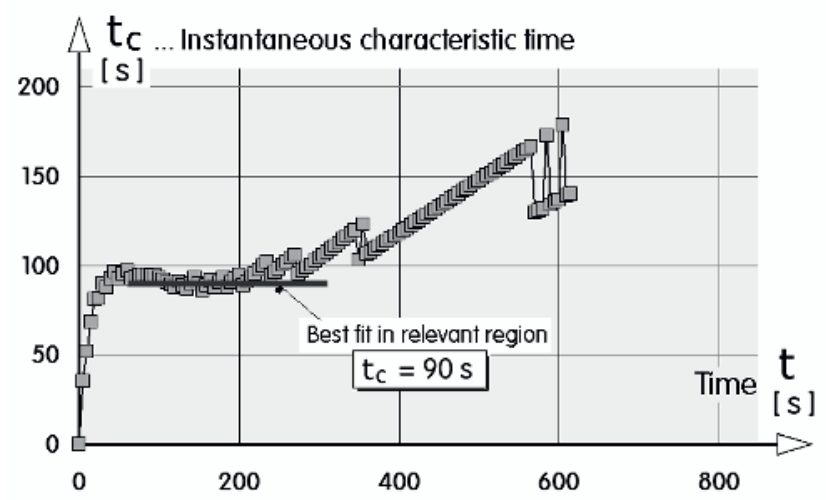

Figure 9. Characteristic time $t_{\mathrm{c}}$ evaluated at individual measurement instants for the data shown in the diagram Figure. 8 , i.e. for the oxygenation with the fluidic generator.

The dynamics of the oxygenation is governed by a slight modification of eq. (8):

$$
\frac{\mathrm{d} m_{\mathrm{O} 2}}{\mathrm{~d} t}=\alpha_{\mathrm{m}} \kappa F\left(m_{\mathrm{O}_{2} \mathrm{~S}}-m_{\mathrm{O}_{2}}\right)
$$

This may be integrated, for the initial condition $m_{\mathrm{O}_{2}}=0$, to

$$
\frac{m_{\mathrm{O}_{2}}}{m_{\mathrm{O}_{2} \mathrm{~S}}}=1-\exp \left(-\frac{t}{t_{\mathrm{c}}}\right)
$$

where the characteristic time is

$$
t_{\mathrm{c}}=1 / \alpha_{\mathrm{m}} \kappa F
$$

an instantaneous value of the characteristic time is evaluated as

$$
t_{c}=\frac{t}{\ln \frac{m_{\mathrm{O}_{2} \mathrm{~S}}}{m_{\mathrm{O}_{2} \mathrm{~S}}-m_{\mathrm{O}_{2}}}}
$$

This was used for plotting the data presented in Figure. 9 for the measurements with the fluidic generator. The values there are not constant. Obviously, the data at the extreme ends of the measurement time are unreliable and could be discarded. The average $t_{\mathrm{c}}=90 \mathrm{~s}$ was thus evaluated only in the really relevant region near the characteristic time, i.e. those obtained near

$$
m_{\mathrm{O}_{2}}=0.632 m_{\mathrm{O}_{2} \mathrm{~S}}
$$

The experiment without the generator too so long time that it was not possible to maintain the required constant conditions. As a result, the measured data deviate quite much from the best fit. Because this experimental run was made only for comparison with the really significant measurements with the generator, this discrepancy was unimportant. Essential fact is the simple end of a tube, still in use in some waste water processing, is many times worse. The value considered to be the best fit there is $t_{\mathrm{c}}=90 \mathrm{~s}$. In other words, the plain tube end, despite the vigorous agitation it produces, needs 4.22 times longer period of time to achieve the same degree of oxygenation.

Another way of evaluating the effectiveness of oxygenation is comparing these measurement results with the $0.128 \mathrm{~g} / \mathrm{s}$ oxygen input flow rate. In the case without the fluidic generator, the 13.8 kilograms of the water in the test vessel need $0.086 \mathrm{~g}$ of oxygen to be oxygenated to the concentration defined in eq. (13). Without the fluidic generator it was necessary to run the aeration for $380 \mathrm{~s}$ delivering the total of 48.6 grams of oxygen, i.e. with efficiency $0.176 \%$. With the generator sufficed for the same task 11.5 grams and the efficiency was $0.74 \%$. However much better this value is, it still shows how large field is there for further improvements.

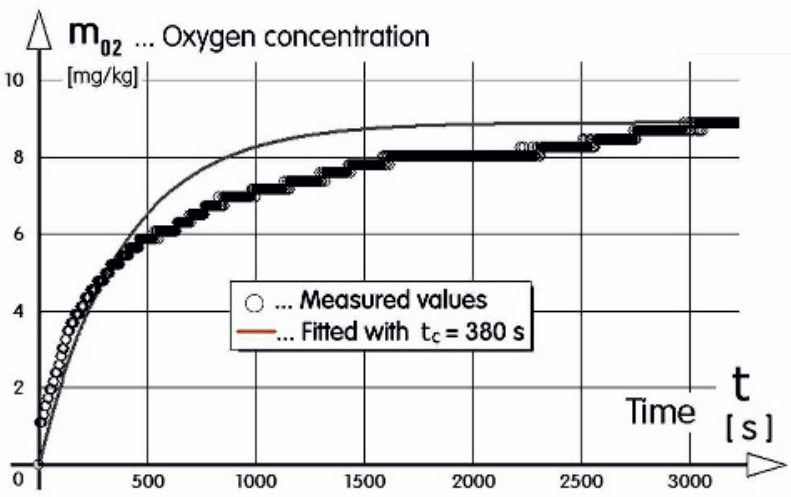

Figure 10. For comparison, the concentration of oxygen $m_{\mathrm{O}_{2}}$ was also measured without the generator at the same supplied air flow rate $0,61 \mathrm{~g} / \mathrm{s}$ - with just $7 \mathrm{~mm}$ inner diameter air supply tube submerged to $170 \mathrm{~mm}$ depth under the water level in the vessel. The transport was so slow (of the order of hours) that it became impossible to maintain constant conditions. 


\section{Conclusions}

A new fluidic generator, with the supplied air flow pulsated by a fluidic oscillator located in the same submersible body as the aerator, was tested by the dynamic oxygenation method [5], measuring the oxygen concentration in the water into which the air bubbles were blown. The generator demonstrated 4.22-times improvement in oxygenation efficiency when compared with the aeration from a plain end of the $7 \mathrm{~mm}$ internal diameter plain supply tube. Despite this significant improvement, the behaviour of the generator still has lot to be desired and can be further improved.

\section{Acknowledgments}

The first author (V. T.) obtained support by grant 1323046S from GAČR, by grant TA02020795 provided by TAČR, and institutional support RVO:61388998.. Dr. J. Hykl made for us the necessary chemistry calculations and handled the elimination of dissolved oxygen between the experimental runs. The electronics for data storage and presentation on the monitor was developed by Mr. M. Pavelka.

\section{References}

1. W.B. Zimmerman, V. Tesař, Bubble generation for aeration and other purposes, British Patent GB20060021561, Filed Oct. 2006

2. V. Tesař, M. Jílek, Proc. of Colloq. 'Fluid dynamics 2013 (2013)

3. V. Tesař, Proc. of Flucome 2013, the $12^{\text {th }}$ Int. Symp. on Fluid Control, Measurement and Visualization, (2013)

4. V. Tesař, Mezní vrstvy a turbulence( Boundary Layers and Turbulence - in Czech), textbook for engineering students, ČVUT Praha 1991

5. P. Juárez, J. Orejach, Latin American Applied Res. 31, $433(2011)$

6. V. Tesař, C.-H. Hung, W. Zimmerman, Sensors and Actuators A: Physical 125, 159 (2006)

7. V. Tesař, Sensors and Actuators A: Physical 138, 394 (2007)

8. V. Tesař, S. Zhong, R. Fayaz, AIAA Journal 51, 397, (2013)

9. V. Tesař, Chemical Engineering Journal 155, 789 (2009)

10. V. Tesař V., Sensors and Actuators A: Physical 138, 394 (2007)

11. V. Tesař, Chemical Engineering Journal 231, 526 (2013)

12 V. Tesař V., WIT Transactions on Engineering Sciences 79, 411 (2013)

13 V. Tesar̆, Proc. of $27^{\text {th }}$ Symp., 62, (2013)

14. V. Tesař, Shape oscillation of microbubbles, Chemical Engineering Journal, in Press (2013)
15. P. Doudoroff, D.I. Shumway, Fisheries and Aquaculture Dept. Fish. Tech. Pap. 86 (1970)

16. W. B. Zimmerman et al., Current Opinion in Colloid and Interface Science 16, 350 (2011)

17. W.B. Zimmerman et al., Food and Bioproducts Processing 87, 215 (2009) 